Editor's Note: These short, critical reviews of recent papers in the Journal, written exclusively by graduate students or postdoctoral fellows, are intended to summarize the important findings of the paper and provide additional insight and commentary. For more information on the format and purpose of the Journal Club, please see http://www.jneurosci.org/misc/ifa_features.shtml.

\title{
Neuronal Degeneration in a Viral Model of Multiple Sclerosis
}

\author{
Raphael Schneider \\ Département de microbiologie et immunologie, Université de Montréal, Notre-Dame Hospital, Montreal, Quebec H2L 4M1, Canada \\ Review of Das Sarma et al.
}

Ever since multiple sclerosis (MS) pathology was defined at the end of the 19 th century, it has been debated whether the primary event in the CNS affects myelin or axons. Even though the characteristic pattern of tissue damage was noticed to be demyelination, it was proposed early on that axonal damage may in fact be responsible for permanent disability in MS patients (Charcot, 1880). Interestingly, axonal injury in MS is not limited to the demyelinated lesions, but occurs even in so-called normal appearing brain tissue (Bitsch et al., 2000), supporting the notion that damaged neurons in non-demyelinated areas contribute to the functional deficits in MS.

Numerous investigations have extended our knowledge of MS pathology using virusinduced models of chronic demyelination. Probably the most extensively studied model is the Theiler's murine encephalomyelitis virus (TMEV), which induces a chronic progressive demyelinating disease in susceptible mice. Although TMEV is cleared from neurons, it persists in glial cells and macrophages subsequently leading to demyelination and axonal injury (for review, see Oleszak et al., 2004). Neurotropic strains of mouse hepatitis virus (MHV) can induce chronic inflammatory demyelina-

Received 0ct. 15, 2009; revised Nov. 10, 2009; accepted Nov. 11, 2009.

This work was supported by a postdoctoral scholarship from the German Academic Exchange Service. I would like to thank my mentor Dr. Nathalie Arbour and also Dr. Patrick Dion for helpful discussion on the manuscript.

Correspondence should be addressed to Raphael Schneider, Département de microbiologie et immunologie, Université de Montréal, NotreDame Hospital, Pavilion JA DeSeve (Y-3609), 1560 Sherbrooke E, Montreal, QC H2L 4M1, Canada. E-mail: raphael.schneider@umontreal.ca.

DOI:10.1523/JNEUROSCI.5198-09.2009

Copyright $\odot 2009$ Society for Neuroscience $\quad$ 0270-6474/09/2915353-02\$15.00/0 tion bearing similar histological features to those observed in MS: lesions associated with lipid-laden macrophages, infiltrating $\mathrm{T}$ lymphocytes and naked axons scattered throughout the CNS. Similarly to TMEV, MHV antigens are detected in astrocytes, oligodendrocytes, and neurons early on, and at later time points can only be found in glial cells (for review, see Matthews et al., 2002).

Das Sarma et al. (2009) addressed the question whether axonal damage occurs as a consequence of direct viral attack or secondary to demyelination in the MHV model. They compared the neuropathological features induced upon infection of C57BL/6 mice with two distinct MHV strains. The demyelinating strain RSA59 (DM) led to demyelination with concomitant axonal injury while the non-demyelinating strain RSMHV2 (NDM) caused focal axonal degeneration without demyelination. The two strains carry the same genomic backbone except for the spike glycoprotein, a structural protein responsible for virushost attachment.

Histopathologically, DM mice displayed large demyelinating plaques on day 7 after infection; on the contrary, infection with NDM left the myelin sheath relatively preserved [Das Sarma et al. (2009), their Figure 2]. Light microscopic and electron micrographic analyses revealed intact naked axons with preserved axolemmas and axoplasm in DM infection but early axonal degeneration with collapse of the myelin sheath in NDM infected animals [Das Sarma et al. (2009), their Figure 3C-D and Figure 4]. One week after intracranial infection with either strain, the majority of inflammatory cells identified in CNS tissue were CD11b + cells (macrophages/microglia) [Das Sarma et al. (2009), their Figure 1 and Figure $6 E-F]$. Macrophage-mediated myelin stripping was seen in DM infection underscoring the role of CD11b + cells in MHV-induced demyelination [Das Sarma et al. (2009), their Figure 5]. Remarkably, although the viral particles were injected directly into the brain, cell infiltrations, primary axonal loss (NDM), and substantial damage to the myelin sheath (DM) were evident in spinal cord sections as early as day 7 postinfection.

Das Sarma et al. (2009) demonstrated that MHV infection can induce inflammation, demyelination, and neurodegeneration resembling MS pathology. Macrophages and activated microglia, abundantly found in MS lesions, have been shown to play key roles in MS pathogenesis. In fact, the number of injured axons in MS lesions correlates with the number of macrophages/microglia present onsite (Trapp et al., 1998). One has to mention that various other inflammatory cells are thought to play crucial roles in myelin and axonal loss. Aberrant activation of auto-reactive CD4 and CD8 T lymphocytes targeting epitopes of the CNS are observed in MS (Sospedra and Martin, 2005). CD4 and CD8 T lymphocytes and their secreted mediators are found in MS lesions, and published evidence suggests that CD8 T lymphocytes have the capacity to cause axonal damage in MS (Bitsch et al., 2000). Das Sarma et al. (2009) did not provide quantification of infiltrating CD4 or CD8 $\mathrm{T}$ lymphocytes in DM or NDM infected animals, thus we cannot estimate nor compare their contribution to tissue injury in these two distinct MHV models. 
Das Sarma et al. (2009) observed different patterns of leukocyte distribution in the brain and spinal cord depending on the viral strain used for induction and the time point studied. At day 7 postinfection, NDM-infected animals displayed leukocytes predominately in the gray matter. In contrast, DM infection led to infiltrated leukocytes restricted to the white matter [Das Sarma et al. (2009), their Figure $6 C, D]$. On day 30 postinfection only a small population of leukocytes was observed in NDM infection, whereas the DM infected mice still showed cell enrichment in demyelinated lesions [Das Sarma et al. (2009), their Figure 7C,D]. These differences in cell location led to the hypothesis that the infiltrating immune cells parallel viral antigen distribution within the CNS. Fluorescence microscopic analysis on day 7 postinfection revealed viral antigen of DM-infected mice predominantly in the white matter, but restriction of viral antigen to the gray matter in NDM-infected animals [Das Sarma et al. (2009), their Figure $8 A, C$ ]. It would have been relevant to assess leukocyte infiltration and viral antigen on the same sections to demonstrate that infiltrating immune cells are indeed migrating toward infected cells. Finally, DM or NDM infection of primary neuronal cultures showed viral antigen spreading in DM-infected cultures, whereas in NDM-infected cultures viral particles seemed to be limited to previously infected neurons [Das Sarma et al. (2009), their Figure 8H,I]. These experiments demonstrate antigen distribution shortly after infection; unfortunately, a quantification of viral spread has not been provided and thus no investigation of viral replication in neurons of the two strains could be made to further complement their findings.

Das Sarma et al. (2009) concluded that DM and NDM strains translocate differently along axons, consequently leading to characteristic patterns of leukocyte distribution in either infection. Plausible explanations mentioned by the authors are NDM transport failure or impaired capacity to infect oligodendrocytes via direct cell-cell contact, accounting for the limited spread of viral particles. As mentioned above, the two viral strains have a different spike glycoprotein, which is responsible for the virus-host attachment and therefore can drastically influence the viral spread and tropism. No analyses over longer time periods were shown; something which might have provided clues as to where the different viral strains can persist. Moreover, the authors also did not report any clinical symptoms despite the observed CNS pathology. The reason for this omission could, however, be that it is difficult to assess the consequences of gray matter involvement in animal models of MS as these insults poorly correlate with physical findings but rather manifest as cognitive dysfunction. One might speculate that DM-infected animals displayed physical disability due to massive demyelination and concomitant axonal damage of ascending and descending fiber tracts, whereas local axonal injury without demyelination as observed in NDM-infection did probably not affect the animals to a measurable extent. Nevertheless, to determine the clinical impact of axonal loss and also possible late-onset findings, it would have been worthwhile to extend the observation period to several months.

In conclusion, Das Sarma et al. (2009) describe a model of non-demyelinating MHV infection (RSMHV2) useful for further study of primary axonal damage in a mouse model of MS. Axonal degeneration was even observed with minor cell infiltration; therefore, NDM-infection of RAG knock-out mice lacking both B and $\mathrm{T}$ lymphocytes could provide further insight into non-immune-mediated axonal injury.
Extensive axonal damage has been reported in the early phase of MS, and even outside demyelinated areas, questioning the widely accepted concept that injury to myelin precedes axonal damage (Filippi et al., 2003).

Whether the demyelination and the neurodegeneration observed in MS are timely distinct or concurrent and colocalized events remains to be determined. As axonal loss is associated with permanent functional impairment in MS, neuroprotective treatment strategies are needed. A precondition for their design, however, is thorough studies of neuropathological mechanisms in animal models such as the one described here.

\section{References}

Bitsch A, Schuchardt J, Bunkowski S, Kuhlmann T, Brück W (2000) Acute axonal injury in multiple sclerosis. Correlation with demyelination and inflammation. Brain 123:11741183.

Charcot JM (1880) Lecons sur les maladies du systeme nerveux faites a la Salpetriere, $4 \mathrm{Ed}$. Paris: Cerf et fils.

Das Sarma J, Kenyon LC, Hingley ST, Shindler KS (2009) Mechanisms of primary axonal damage in a viral model of multiple sclerosis. J Neurosci 29:10272-10280.

Filippi M, Bozzali M, Rovaris M, Gonen O, Kesavadas C, Ghezzi A, Martinelli V, Grossman RI, Scotti G, Comi G, Falini A (2003) Evidence for widespread axonal damage at the earliest clinical stage of multiple sclerosis. Brain 126:433-437.

Matthews AE, Weiss SR, Paterson Y (2002) Murine hepatitis virus-a model for virus-induced CNS demyelination. J Neurovirol 8:76-85.

Oleszak EL, Chang JR, Friedman H, Katsetos CD, Platsoucas CD (2004) Theiler's virus infection: a model for multiple sclerosis. Clin Microbiol Rev 17:174-207.

Sospedra M, Martin R (2005) Immunology of multiple sclerosis. Annu Rev Immunol 23:683747.

Trapp BD, Peterson J, Ransohoff RM, Rudick R, Mörk S, Bö L (1998) Axonal transection in the lesions of multiple sclerosis. N Engl J Med 338:278-285. 